\title{
Synthesis and surface active properties of cationic surface active agents from crude rice bran oil
}

\author{
By W. I. A. El-Dougdoug
}

Chemistry Department, Faculty of Science, Zagazig University, Benha Branch, Benha Egypt.

\section{RESUMEN}

Síntesis y propiedades tensioactivas de agentes tensioactivos catiónicos de aceite de germen de arroz crudo.

Se han preparado tensioactivos catiónicos de hidróxidos del 2-hidroxi-3-(2-alquilamidopolietilamino) propano-1-trietilamonio (ixxiii) $)_{\mathrm{a}-\mathrm{d}}$ a partir de los ácidos grasos (i) a-d [ácido palmítico, esteárico, oleico y linoleico] y mezclas de ácidos grasos de aceite de germen de arroz crudo ie [RBO]. La reacción de estos ácidos con etilenodiamina, dietilenotriamina, trietilenotetramina y tetraetilenopentamina (ii) $)_{a-d}$ produjo los compuestos (iii-vii) $)_{a-d}$ Los amidopolietilaminos producidos (iii-vii) a-d reaccionaron con el cloruro de 2-epoxipropilenotrietilamonio (viii) para dar los tensioactivos catiónicos (ix-xiii) $)_{a-d}$. Los derivados producidos se purificaron y caracterizaron por microanálisis, determinación del peso molecular espectros de infrarrojo (IR) y resonancia magnética nuclear de protón ('H NMR). Se determinaron las propiedades tensioactivas y la eficacia de inhibición de los tensioactivos catiónicos preparados.

PALABRAS-CLAVE: Aceite de germen de arroz crudo - Propiedades tensioactivas - Síntesis - Tensioactivo catiónico.

\section{SUMMARY}

Synthesis and surface active properties of cationic surface active agents from crude rice bran oil.

Cationic surfactants of 2-hidroxy-3-(2-alkylamidopolyethyl amino) propane-1-triethylammonium hydroxides (ix-xiii)a-d were prepared from fatty acids $(i)_{a-d}$ [palmitic, stearic, oleic, linoleic acid] and mixed fatty acids of crude rice bran oil $i_{e}[R B O]$. The reaction of these acids with ethylenediamine, diethylenetriamine, triethylenetetramine and tetraethylenepentamine (ii) a-d produced (iii-vii) $)_{\text {a-d. }}$. The produced amidopolyethylamine $(\text { iii-vii })_{\text {a-d }}$ reacted with 2-epoxypropylenetriethylammonium chloride (viii) to give the

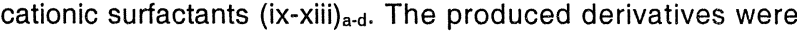
purified and characterized by microanalysis, molecular weight determination, infra-red (IR), and proton nuclear magnetic resonance ( ${ }^{1} \mathrm{H}$ NMR) spectra. The surface active properties and inhibition efficiency of the prepared cationic surfactants were determined.

KEY-WORDS: Cationic surfactant - Crude rice bran oil - Surface active properties - Synthesis.

\section{INTRODUCTION}

It has been reported that cationic surfactants, especially those having quaternary ammonium groups, display an important role in the textile industry. They have antimicrobial activities and emulsifying properties in addition to their anticorrosive effects (1-8). These facts encouraged us to prepare a series of novel groups of cationic surfactants from the mixed fatty acids of egyptian crude rice bran oil, which expected to have good emulsifying properties in nonedible medium as insecticides, and pesticides. The prepared surface active agent has the following formula.

$$
\begin{aligned}
& \mathrm{R}-\mathrm{CO}-\mathrm{NH}-\left[\mathrm{CH}_{2}-\mathrm{CH}_{2}-\mathrm{NH}\right]_{n}-\mathrm{CH}_{2}-\underset{\text { (ix - xiii) }}{\mathrm{CH}-\mathrm{OH}}- \\
& \mathrm{CH}_{2}-\mathrm{N}^{+} \mathrm{Et}_{3} \mathrm{OH}-
\end{aligned}
$$

R -

n

$\mathrm{CH}_{3}-\left(\mathrm{CH}_{2}\right)_{14}$ - (palmitic) 1

$\mathrm{CH}_{3}-\left(\mathrm{CH}_{2}\right)_{16}$ - (stearic) 2

$\mathrm{CH}_{3}-\left(\mathrm{CH}_{2}\right)_{7}-\mathrm{CH}=\mathrm{CH}-\left(\mathrm{CH}_{2}\right)_{7}-$ (oleic) 3

$\mathrm{CH}_{3}-\left(\mathrm{CH}_{2}\right)_{4}-\mathrm{CH}=\mathrm{CH}-\mathrm{CH}_{2}-\mathrm{CH}=\mathrm{CH}-\left(\mathrm{CH}_{2}\right)_{7}$ - (linoleic) 4

Mixed alkyl of rice bran fatty acid composition.

The structure of these compounds was based on the presence of hydrophobic part (fatty acids of rice bran oil) and hydrophilic part (quaternary ammonium moiety, amidopolyethylamino in addition to hydroxyl groups).

\section{MATERIAL AND METHODS}

All melting points are uncorrected; microanalysis and molecular weight determination by osmometric method, using $\mathrm{CHCl}_{3}$ as solvent; IR spectrum were measured by a Pye Unicam SR-1000 infrared spectrophotometer as Nujul mul and ${ }^{1} \mathrm{H}$ NMR spectra on a Varian EM-390 spectrophotometer operating at $90 \mathrm{MHz}$, using $\left(\mathrm{CD}_{3}\right)_{2} \mathrm{CO}$ as solvent and tetramethylsilane (TMS) as internal standard have been done.

\subsection{Crude Rice Bran Oil}

The oil was supplied by the Alexanderia company for extraction and hydrogenation of oils. The specifications are given in (Table I). 
Table I

Specification of crude rice bran oil

\begin{tabular}{lr}
\hline Saponification value & 179 \\
lodine value & 108 \\
Acid value & 68 \\
\hline
\end{tabular}

\subsection{Hydrolysis of Crude Rice Bran Oil}

The procedure described by El-Sawy (9) was followed. The fatty acid mixture was analysed by G.L.C and its composition is given in (Table II).

Table II

Fatty acids composition of rice bran oil

\begin{tabular}{lc}
\hline \multicolumn{1}{c}{ Acid } & Peak area \% \\
\hline Saturated fatty acids & \\
Palmitic & 17.6 \\
Stearic & 00.9 \\
Unsaturated fatty acids & \\
Oleic & 50.1 \\
Linoleic & 27.1 \\
Linolenic & 02.2 \\
\hline
\end{tabular}

The oleic acid is the main constituent of mixed fatty acids of RBO; the molecular weight is considered as oleic $\operatorname{acid}_{\left(\mathrm{M} . \mathrm{W}_{\mathrm{t}}=282\right) \text {. }}$.

\subsection{N-Acylamidopolyethylamino Derivatives (iii-vii)a-d}

N-Pentadecyl-, heptadecyl-, heptadec-9-enyl-, heptadec-9, 12-dienyl and mixed acyl of RBO fatty acids amidopolyethylamino derivatives (iii-vii) $)_{a-d}$ were prepared by condensation of equimolar amounts of the corresponding pure fatty acid (palmitic, stearic, oleic and linoleic acid) or mixed fatty acids from rice bran oil with ethylenediamine, diethylenetriamine, triethylenetetramine or tetraethylenepentamine (ii) a-d. The reaction mixture was heated at $140{ }^{\circ} \mathrm{C}$ for 10 hours. The liberated theoretical amount of water was collected to ensure the reaction completion, by using Dean and Stark apparatus and benzene as solvent. The organic solvent was removed under reduced pressure, and the residue was recrystallized from isopropanol. The yields, melting points, and elemental analysis were used to identify the obtained compounds and collected in Table III.

\subsection{2,3-Epoxypropyl triethylammonium Chloride (viii)}

A mixture of $(9.25 \mathrm{~g}, 0.1$ mole) epichlorohydrin and $(10.19 \mathrm{~g}, 0.1$ mole) triethylamine in $10 \mathrm{ml}$ of distilled water was stirred at room temperature for about 5.5 hours. The resulting clear solution which consists mainly of compound (viii) was used directly in the next step without further purification (10).

\subsection{Conversion of the Prepared $\mathrm{N}$-acylamidopolyethylamino Derivatives to Cationic Surfactants (ix-xiii)a-d}

A mixture of $(0.1 \mathrm{~mol}$.) from the fatty amidopolyethylamino derivatives (iii-vii)a-d and an adequate amount of 2,3-epoxypropylammonium chloride (viii) in $100 \mathrm{ml}$ dry benzene, was stirred under reflux for about 24 hours. The solvent was evaporated, and the remaining waxy residue was thoroughly extracted with hot distilled water till no froth persisted. The aqueous extract was subjected to high speed centrifugation $\left(10^{4} \mathrm{rpm}\right)$ for $15 \mathrm{~min}$ to remove the suspended particles of the unreacted matter. The supernatant clear aqueous layer was decanted, shaken with pet. ether $\left(40-60{ }^{\circ} \mathrm{C}\right)$ to remove the residual unreacted matter, and rendered alkaline with $10 \%$ sodium hydroxide solution. The product was extracted three times each with $50 \mathrm{ml}$ portions of isoamyl alcohol (11). The solvent was evaporated on a boiling water-bath under vacuum to leave a pale yellow waxy residue (cf. scheme 1). Elemental analysis; molecular weight determination, IR and ${ }^{1} \mathrm{H}$ NMR spectroscopic analysis were conducted to confirm the structure of some examples of the prepared compounds (cf. Tables IV-V).

\subsection{Evaluation of the Surface Active Properties of the Prepared Cationic Surfactants}

2.6.1. Surface and interfacial tensions, were measured using a Du-Nouy tensiometer (12) [Kruss, Type 8451] with $0.1 \mathrm{wt} \%$ aqueous solution at room temperature $25^{\circ} \mathrm{C}$.

2.6.2. Kraft point, of the prepared surfactants, were measured as the temperature at which $1 \%$ dispersion becomes clear on gradual heating (13).

2.6.3. Cloud point, was determined by gradually heating $1 \mathrm{wt} \%$ solution in controlled temperature bath and recoding the at which the clear, or nearly clear, solutions become definitely turbid. The reproducibility of this temperature was checked by cooling the solutions until they become clear again (14). 
Table III

Elemental analysis of $\mathrm{N}$-acylamido polyethylamino derivatives (iii-vii)a-d $\mathrm{R}-\mathrm{CO}-\mathrm{NH}-\left(\mathrm{CH}_{2}-\mathrm{CH}_{2} \mathrm{NH}-\right)_{\mathrm{n}}-\mathrm{H}$

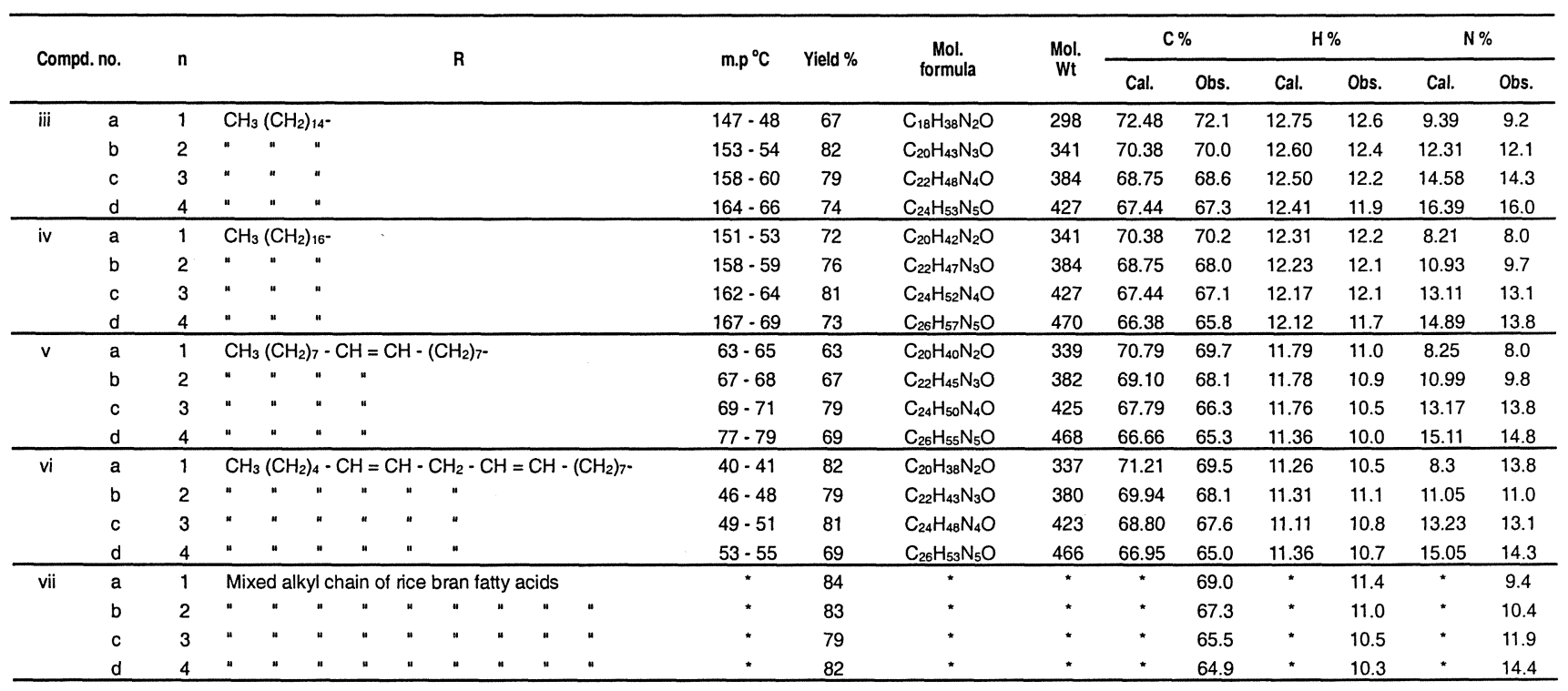

* Not calculated because is the mixed fatty acids composition of crude rice bran oil.

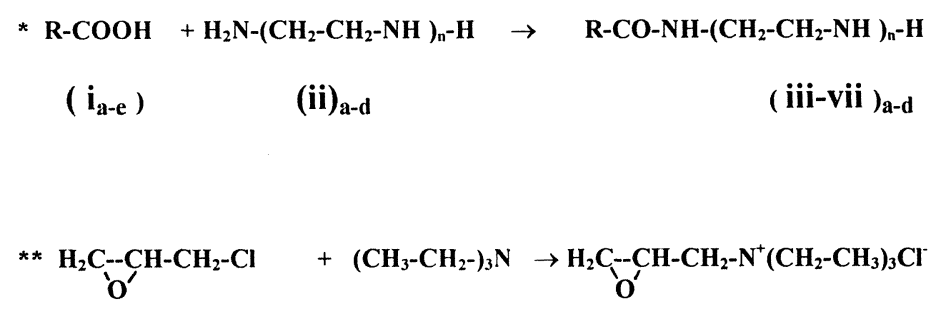

(viii)

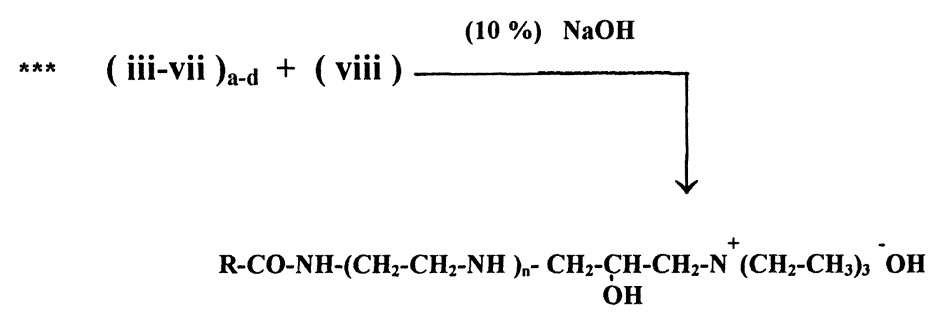

( ix- xiii $)_{a-d}$

Where:

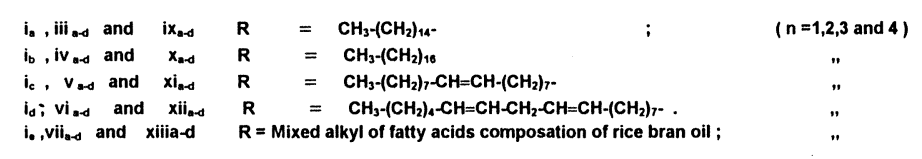

Scheme 1 
Table IV

Elemental analysis of the prepared surfactants (ix - xiii)a-d $\mathrm{R}-\mathrm{CO}-\mathrm{NH}\left(-\mathrm{CH}_{2}-\mathrm{CH}_{2} \mathrm{NH}-\right)_{\mathrm{n}} \mathrm{CH}_{2}-\mathrm{CH}(\mathrm{OH})-\mathrm{CH}_{2}-\mathrm{N}^{+} \mathrm{Et}_{3}-\mathrm{OH}$

\begin{tabular}{|c|c|c|c|c|c|c|c|c|c|c|c|c|c|c|c|c|c|}
\hline \multirow{2}{*}{\multicolumn{2}{|c|}{ Compd. no. }} & \multirow{4}{*}{$\begin{array}{l}n \\
1 \\
2\end{array}$} & \multirow{2}{*}{\multicolumn{4}{|c|}{$\mathbf{R}$}} & \multirow{3}{*}{$\frac{\mathrm{m} \cdot \mathrm{p}^{\circ} \mathrm{C}}{\text { waxy }}$} & \multirow{3}{*}{$\begin{array}{c}\text { Yield \% } \\
28\end{array}$} & \multirow{3}{*}{$\begin{array}{c}\begin{array}{c}\text { Mol. } \\
\text { formula }\end{array} \\
\mathrm{C}_{27} \mathrm{H}_{59} \mathrm{~N}_{3} \mathrm{O}_{3} \cdot 2 \mathrm{H}_{2} \mathrm{O}\end{array}$} & \multicolumn{2}{|c|}{ Mol. Wt ** } & \multicolumn{2}{|c|}{$C \%$} & \multicolumn{2}{|c|}{$\mathrm{H} \%$} & \multicolumn{2}{|c|}{$\mathrm{N} \%$} \\
\hline & & & & & & & & & & \multirow{2}{*}{$\frac{\text { Cal. }}{509}$} & \multirow{2}{*}{$\frac{\text { Obs. }}{500}$} & \multirow{2}{*}{$\frac{\text { Cal. }}{63.65}$} & \multirow{2}{*}{$\frac{\text { Obs. }}{62.1}$} & \multirow{2}{*}{$\frac{\text { Cal. }}{12.37}$} & \multirow{2}{*}{$\frac{\text { Obs. }}{12.0}$} & \multirow{2}{*}{$\frac{\text { Cal. }}{8.25}$} & \multirow{2}{*}{$\frac{\text { Obs. }}{8.4}$} \\
\hline ix & a & & $\mathrm{CH}_{3}(\mathrm{C}$ & & & & & & & & & & & & & & \\
\hline & b & & " " & $"$ & & & waxy & 22 & $\mathrm{C}_{29} \mathrm{H}_{64} \mathrm{~N}_{4} \mathrm{O}_{3} .2 \mathrm{H}_{2} \mathrm{O}$ & 552 & 568 & 63.3 & 63.0 & 11.59 & 11.4 & 10.15 & 10.1 \\
\hline & c & 3 & $"$ & $"$ & & & waxy & 28 & $\mathrm{C}_{31} \mathrm{H}_{69} \mathrm{~N}_{5} \mathrm{O}_{3} .2 \mathrm{H}_{2} \mathrm{O}$ & 595 & 540 & 62.52 & 61.6 & 11.59 & 11.2 & 11.76 & 10.6 \\
\hline & d & 4 & $"$ & $"$ & & & visc. oil & 27 & $\mathrm{C}_{33} \mathrm{H}_{74} \mathrm{~N}_{6} \mathrm{O}_{3} .2 \mathrm{H}_{2} \mathrm{O}$ & 638 & 630 & 62.06 & 61.9 & 11.60 & 11.0 & 13.16 & 13.0 \\
\hline \multirow[t]{4}{*}{$x$} & $a$ & 1 & $\mathrm{CH}_{3}(\mathrm{C}$ & & & & waxy & 27 & $\mathrm{C}_{29} \mathrm{H}_{63} \mathrm{~N}_{3} \mathrm{O}_{3} .2 \mathrm{H}_{2} \mathrm{O}$ & 537 & 528 & 64.8 & 64.0 & 12.19 & 12.2 & 7.82 & 8.0 \\
\hline & $b$ & 2 & " " & $"$ & & & waxy & 29 & $\mathrm{C}_{31} \mathrm{H}_{68} \mathrm{~N}_{4} \mathrm{O}_{3} \cdot 2 \mathrm{H}_{2} \mathrm{O}$ & 580 & 570 & 64.13 & 63.9 & 11.68 & 12.1 & 9.65 & 9.3 \\
\hline & c & 3 & $"$ & $"$ & & & waxy & 32 & $\mathrm{C}_{33} \mathrm{H}_{73} \mathrm{~N}_{5} \mathrm{O}_{3} \cdot 2 \mathrm{H}_{2} \mathrm{O}$ & 623 & 620 & 63.56 & 62.8 & 11.70 & 12.1 & 11.25 & 10.1 \\
\hline & d & 4. & " " " & $"$ & & & waxy & 30 & $\mathrm{C}_{35} \mathrm{H}_{78} \mathrm{~N}_{6} \mathrm{O}_{3} .2 \mathrm{H}_{2} \mathrm{O}$ & 666 & 645 & 63.06 & 62.0 & 11.70 & 11.7 & 12.61 & 12.8 \\
\hline \multirow[t]{4}{*}{$x i$} & a & 1 & $\mathrm{CH}_{3}(\mathrm{C}$ & $7-$ & $\mathrm{H}=\mathrm{CH}$ & $\left.\mathrm{CH}_{2}\right)_{7}$ & visc. oil & 23 & $\mathrm{C}_{29} \mathrm{H}_{61} \mathrm{~N}_{3} \mathrm{O}_{3} .2 \mathrm{H}_{2} \mathrm{O}$ & 535 & 536 & 65.04 & 64.7 & 11.40 & 11.0 & 7.85 & 8.0 \\
\hline & $\mathrm{b}$ & 2 & " $\quad "$ & $"$ & $"$ & & visc. oil & 21 & $\mathrm{C}_{31} \mathrm{H}_{66} \mathrm{~N}_{4} \mathrm{O}_{3} \cdot 2 \mathrm{H}_{2} \mathrm{O}$ & 580 & 570 & 64.35 & 64.3 & 11.41 & 10.9 & 9.68 & 9.1 \\
\hline & c & 3 & $"$ & $"$ & $"$ & & visc. oil & 29 & $\mathrm{C}_{33} \mathrm{H}_{71} \mathrm{~N}_{5} \mathrm{O}_{3} \cdot 2 \mathrm{H}_{2} \mathrm{O}$ & 621 & 612 & 63.76 & 62.1 & 11.43 & 10.5 & 11.27 & 11.0 \\
\hline & d & 4 & " " & $"$ & " & & visc. oil & 26 & $\mathrm{C}_{35} \mathrm{H}_{76} \mathrm{~N}_{6} \mathrm{O}_{3} .2 \mathrm{H}_{2} \mathrm{O}$ & 664 & 654 & 63.25 & 63.0 & 11.44 & 10.0 & 12.65 & 12.1 \\
\hline \multirow[t]{4}{*}{ xii } & $a$ & 1 & $\mathrm{CH}_{3}(\mathrm{C}$ & & $=\mathrm{CH}-\mathrm{C}$ & $2-\mathrm{CH}=\mathrm{CH}\left(\mathrm{CH}_{2}\right)$ & visc. oil & 25 & $\mathrm{C}_{29} \mathrm{H}_{59} \mathrm{~N}_{3} \mathrm{O}_{3} \cdot 2 \mathrm{H}_{2} \mathrm{O}$ & 533 & 510 & 65.29 & 63.4 & 11.81 & 10.9 & 7.87 & 7.9 \\
\hline & $\mathrm{b}$ & 2 & " " & $"$ & " " & " & visc. oil & 26 & $\mathrm{C}_{31} \mathrm{H}_{64} \mathrm{~N}_{4} \mathrm{O}_{3} \cdot 2 \mathrm{H}_{2} \mathrm{O}$ & 576 & 546 & 64.58 & 63.5 & 12.15 & 11.9 & 9.72 & 8.9 \\
\hline & c & 3 & $"$ & $"$ & $"$ & $"$ & visc. oil & 20 & $\mathrm{C}_{33} \mathrm{H}_{69} \mathrm{~N}_{5} \mathrm{O}_{3} \cdot 2 \mathrm{H}_{2} \mathrm{O}$ & 619 & 593 & 63.97 & 61.8 & 11.79 & 11.8 & 11.30 & 10.9 \\
\hline & $d$ & 4 & $"$ & $"$ & $"$ & $"$ & visc. oil & 28 & $\mathrm{C}_{35} \mathrm{H}_{74} \mathrm{~N}_{6} \mathrm{O}_{3} \cdot 2 \mathrm{H}_{2} \mathrm{O}$ & 662 & 618 & 63.44 & 62.1 & 11.78 & 10.0 & 12.68 & 12.3 \\
\hline \multirow[t]{4}{*}{ xiii } & a & 1 & Mixed & $\mathrm{ylc}$ & in of ric & ran fatty acids & waxy & 29 & $*$ & $\star$ & * & $*$ & 64.4 & * & 11.9 & $*$ & 8.3 \\
\hline & b & 2 & " " & $"$ & " " " & & waxy & 31 & * & * & * & * & 55.9 & $*$ & 11.0 & * & 9.4 \\
\hline & c & 3 & $"$ & $"$ & $"$ & & waxy & 29 & * & * & * & $*$ & 63.2 & * & 10.6 & * & 10.9 \\
\hline & d & 4 & $"$ & $"$ & $"$ & & visc. oil & 34 & * & * & $*$ & * & 61.4 & * & 10.7 & * & 11.4 \\
\hline
\end{tabular}

* Not calculated because is the mixed fatty acids composition of crude rice bran oil.

** Allowable error of the mol. weight determination by osmometric method is $10 \%$.

Table V

Spectral data of some examples from the prepared surfactants $\mathrm{R}-\mathrm{CO}-\mathrm{NH}\left(-\mathrm{CH}_{2}-\mathrm{CH}_{2} \mathrm{NH}-\right)_{\mathrm{r}}{ }^{-} \mathrm{CH}_{2}-\mathrm{CH}(\mathrm{OH})-\mathrm{CH}_{2}-\mathrm{NEt}^{+}{ }_{3} \mathrm{OH} .^{-}$

\begin{tabular}{|c|c|c|}
\hline Compound no. & ${ }^{1} \mathrm{H}$ NMR $(\delta=\mathrm{ppm})$ & $\operatorname{IR}\left(\mathrm{cm}^{-1}\right)$ \\
\hline ix a & $\begin{array}{c}\delta 0.8\left(\mathrm{t}, 3 \mathrm{H} \text {, term. } \mathrm{CH}_{3}\right) ; \delta 0.9 \text { (br. s.; } 26 \mathrm{H}, \mathrm{CH}_{2} \text { chain); } \delta 1.5 \\
\left.\text { (br.t, } 2 \mathrm{H}, \mathrm{CH}_{2} \mathrm{CO}-\right) ; \delta 2.0-2.2 \text { (br.s; } 6 \mathrm{H},-\mathrm{NH} \mathrm{CH}_{2} \mathrm{CH}_{2}-\mathrm{NH}- \\
\mathrm{CH}_{2-}-; \delta 2.6\left(\mathrm{t}, 9 \mathrm{H}, \mathrm{N}\left(-\mathrm{CH}_{2}-\mathrm{CH}_{3}\right)_{3} ; \delta 3.9\left(\mathrm{~m}^{2} 8 \mathrm{H}_{1} \mathrm{CH}_{2} \mathrm{~N}\right.\right. \\
\left.\left(-\mathrm{CH}_{2}-\mathrm{CH}_{3}\right) ; \delta 4.0 \text { (br.s, } 1 \mathrm{H} ; \mathrm{OH}\right) ; \text { and } \delta 7.9 \\
\text { (s.; } 1 \mathrm{H} \text {, ionized OH). }\end{array}$ & $\begin{array}{l}3350-3200 \mathrm{~cm}^{-1} \vee \mathrm{NH} ; 3500-3400 \mathrm{~cm}^{-1} \vee \mathrm{OH} ; 1650-1560 \\
\mathrm{~cm}^{-1} \vee \mathrm{CO} \text { of amide and broad band at; } 2050-2200 \mathrm{~cm}^{-1} \\
\text { for } \vee \mathrm{CH}_{2} \mathrm{~N}^{+}\left(-\mathrm{CH}_{2}-\mathrm{CH}_{3}\right)_{3} .\end{array}$ \\
\hline ix b & $\begin{array}{c}\delta 0.9\left(\mathrm{t}, 3 \mathrm{H} \text {, term. } \mathrm{CH}_{3}\right) ; \delta 1.2 \text { (br. s.; } 26 \mathrm{H}, \mathrm{CH}_{2} \text { chain); } \delta 1.25 \\
\left.\text { (br.t, } 2 \mathrm{H}, \mathrm{CH}_{2} \mathrm{CO}-\right) ; \delta 1.3-1.4 \text { (br.s; } 10 \mathrm{H},-\mathrm{NH} \mathrm{CH}_{2} \mathrm{CH}_{2}-\mathrm{NH} \\
\left.\quad-\mathrm{CH}-2-\mathrm{CH}_{2}-\mathrm{NH}-\mathrm{CH}_{2}-\right) ; \delta 2.8\left(\mathrm{t} ; 9 \mathrm{H}, \mathrm{N}\left(-\mathrm{CH}_{2}-\mathrm{CH}_{3}\right) 3 ;\right. \\
\delta 3.7\left(\mathrm{~m} ; 8 \mathrm{H}, \mathrm{CH}_{2} \mathrm{~N}\left(-\mathrm{CH}_{2}-\mathrm{CH}_{3}\right) 3 ; \text { and } \delta 4.0(\text { br.s, } 1 \mathrm{H} ; \mathrm{OH}) .\right.\end{array}$ & $\begin{array}{c}3400-3500 \mathrm{~cm}^{-1} v \mathrm{OH} ; 3350-3200 \mathrm{~cm}^{-1} v \mathrm{NH} ; 1560 \mathrm{~cm}^{-1} \\
v \mathrm{CO} \text { of amide; } 2100 \mathrm{~cm}^{-1} \text { for } v-\mathrm{CH}_{2} \mathrm{~N}^{+}\left(-\mathrm{CH}_{2}-\mathrm{CH}_{3}\right)_{3} .\end{array}$ \\
\hline $\mathrm{xa}$ & $\begin{array}{c}\left.\delta 0.7 \text { (t,3H, term. } \mathrm{CH}_{3}\right) ; \delta 0.9 \text { (br. s.; } 30 \mathrm{H}, \mathrm{CH}_{2} \text { chain); } \delta 1.5 \\
\left.\text { (br.t, } 2 \mathrm{H}, \mathrm{CH} \mathrm{CH}_{2} \mathrm{CO}-\right) ; \delta 2.0-2.2\left(\text { br.s; } 6 \mathrm{H},-\mathrm{NH}-\mathrm{CH}_{2}-\mathrm{CH}_{2}-\mathrm{NH}-\right. \\
\left.\mathrm{CH}_{2}-\right) ; \delta 2.6\left(\mathrm{t}, 9 \mathrm{H}, \mathrm{N}\left(-\mathrm{CH}_{2}-\mathrm{CH}_{3}\right)_{3} ; \delta 3.9\left(\mathrm{~m}_{3} ; \mathrm{H}_{1}, \mathrm{CH}_{2} \mathrm{~N}\right.\right. \\
\left.\left(-\mathrm{CH}_{2}-\mathrm{CH}_{3}\right)_{3} ; \delta 4.0 \text { (br.s, } 1 \mathrm{H} ; \mathrm{OH}\right) ; \text { and } \delta 7.9(\mathrm{~s} . ; 1 \mathrm{H} ; \mathrm{OH}) \text {. }\end{array}$ & $\begin{array}{c}3400-3500 \mathrm{~cm}^{-1} v \mathrm{OH} ; 3350-3200 \mathrm{~cm}^{-1} v \mathrm{NH} ; 1650 \mathrm{~cm}^{-1} v \\
\mathrm{C}=\mathrm{C} \text { of oleic chain } 1580 \mathrm{~cm}^{-1} v \mathrm{CO} \text { of amide } 2080 \mathrm{~cm}^{-1} \\
\text { for } v \mathrm{CH}_{2} \mathrm{~N}^{+}\left(-\mathrm{CH}_{2}-\mathrm{CH}_{3}\right)_{3} .\end{array}$ \\
\hline xi a & 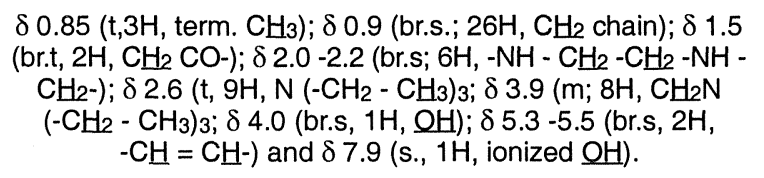 & $\star * * * \star * \star * *$ \\
\hline xii a & 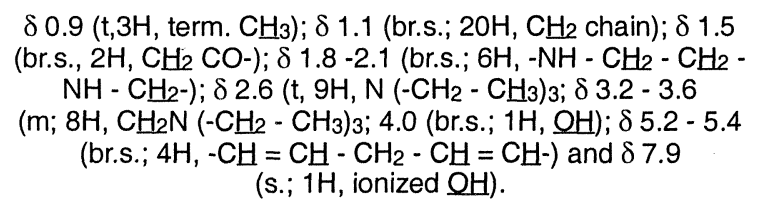 & $\begin{array}{l}3400-3500 \mathrm{~cm}^{-1} v \mathrm{OH}^{-1} 3350-3200 \mathrm{~cm}^{-1} v \mathrm{NH} ; 3100 \mathrm{~cm}^{-1} v \\
\mathrm{CH} \text { - olifinic; } 1650 \mathrm{~cm}^{-1} v \mathrm{C}=\mathrm{C} \text { of oleic chain } 1580 \mathrm{~cm}^{-1} v \\
\quad \text { CO of amid } 2100 \mathrm{~cm}^{-1} \text { for } \mathrm{CH}_{2} \mathrm{~N}^{+}\left(-\mathrm{CH}_{2}-\mathrm{CH}_{3}\right)_{3} .\end{array}$ \\
\hline xiii a & $\begin{array}{c}\left.\delta 0.9 \text { (t,3H, term. } \mathrm{CH}_{3}\right) ; \delta 1.1 \text { (br.s.; } \mathrm{CH}_{2} \text { chain); } 1.4 \\
\left.\text { (br.s., } 2 \mathrm{H}, \mathrm{CH}_{2} \mathrm{CO}-\right) ; \delta 1.9-2.2 \text { (br.s.; } 6 \mathrm{H}_{1}-\mathrm{NH} \mathrm{CH}_{2} \mathrm{CH}_{2}- \\
\left.\mathrm{NH}-\mathrm{CH}_{2}-\right) ; \delta 2.7\left(\mathrm{t}, 9 \mathrm{H}, \mathrm{N}\left(-\mathrm{CH}_{2}-\mathrm{CH}_{3}\right) ; \delta 3.2-3.6\right. \\
\left.\text { (m; } 8 \mathrm{H}, \mathrm{CH}_{2} \mathrm{~N}\left(-\mathrm{CH}_{2}-\mathrm{CH}_{3}\right)_{3} ; \delta 4.0 \text { (br.s.; } 1 \mathrm{H}, \mathrm{OH}\right) ; \delta 5.2- \\
5.4 \text { (br.s, -olifinic protons) and } \delta 7.9 \text { (s.; } 1 \mathrm{H} \text {, ionized } \mathrm{OH}) \text {. }\end{array}$ & $\begin{array}{l}3400-3500 \mathrm{~cm}^{-1} v \mathrm{OH}^{3} 3350-3200 \mathrm{~cm}^{-1} v \mathrm{NH} ; 3100 \mathrm{~cm}^{-1} v \\
\mathrm{CH}-\text { olifinic; } 1650 \mathrm{~cm}^{-1} v \mathrm{C}=\mathrm{C} \text { of unsaturated fatty chain; } \\
1560 \mathrm{~cm}^{-1} \vee \mathrm{CO} \text { of amid } 2080 \mathrm{~cm}^{-1} \text { for } \mathrm{CH}_{2} \mathrm{~N}^{+}\left(-\mathrm{CH}_{2}-\mathrm{CH}_{3}\right)_{3} \text {. }\end{array}$ \\
\hline
\end{tabular}


2.6.4. Wetting time, was determined by immersing a sample of cotton fabric in $0.1 \mathrm{wt} \%$ aqueous solution of the surfactants (15).

2.6.5. Foaming properties, were measured by Ross Miles (16) method. The foam production for 0.1 wt\% solution was measured by the foam height initially produced.

2.6.6. Emulsion stability: The emulsion was prepared from $10 \mathrm{ml}$. of a $20 \mathrm{~m}$ mole aqueous solution of surfactant and $5 \mathrm{ml}$ of toluene at $40{ }^{\circ} \mathrm{C}$. The emulsifying property was determined by the time it took for an aqueous volume separating from the emulsion layer to reach $9 \mathrm{ml}$ counting from the moment of the cession shaking (17).

2.6.7 Stability to hydrolysis: a mixture of $10 \mathrm{~m}$ mole surfactant and $10 \mathrm{ml} 2 \mathrm{~N} \mathrm{H}_{2} \mathrm{SO}_{4}$ or $0.05 \mathrm{~N}$ $\mathrm{NaOH}$ were placed in a thermostat at $40^{\circ} \mathrm{C}$. The time it takes for a sample solution to be clouded as a result of hydrolysis shows the stability of surfactant to hydrolysis (18).

\subsection{Inhibition Efficiency}

The effect of increasing concentrations for some prepared cationic surfactants as corrosion inhibitors for dissolution of carbon steel (Type $5 \mathrm{Px}-L$ ) was studied using weight loss technique (19). The chemical composition of carbon steel $0.26 \%$ C, $1.35 \% \mathrm{Mn}, 0.09 \% \mathrm{P}, 0.05 \% \mathrm{~S}, 0.005 \mathrm{Nb}, 0.02 \%$ $\mathrm{V}, 0.03 \% \mathrm{Ti}$ and the remainder is iron. Carbon-steel compositions measuring $10 \times 20 \times 3 \mathrm{~mm}$ were used. The specimen was immersed in $100 \mathrm{ml}$ of test solution for period of 6 hours at room temperature.
The percent of inhibition efficiency (I.E) was calculated from the following equation:

$$
\text { I.E }=100\left[1-\left(W_{\text {add }} / W_{\text {free }}\right)\right]
$$

Where: $W_{\text {add }}$ and $W_{\text {free }}$ are the weight loss of steel in presence and in absence of the inhibitor.

\section{RESULTS AND DISCUSSION}

Preparation of cationic surfactants (ix-xiii) $)_{a-d}$ was easy and could be isolated in suitable yield (cf. Tables III-IV); infra red (IR) and proton nuclear magnetic resonance ( ${ }^{1} \mathrm{H}$ NMR) spectroscopic analysis were conducted to confirm the structure of some examples of the prepared compounds (cf. Table V).

3.1. Surface and interfacial tension: Cationic surfactants prepared from unsaturated acids (xi-xii) $)_{\text {a-d }}$ recorded higher surface and interfacial tension than those prepared from saturated fatty acids (ix- $\mathrm{x})_{\mathrm{a}-\mathrm{d}}$ (cf. Tables VI-VII); while surfactants obtained from mixed fatty acids of crude rice bran oil recorded almost the same surface and interfacial tension as those prepared from individual commercial unsaturated fatty acid (cf. Table VIII). Also, it was noted that as the ethylamino unit increased the surface and interfacial tension decreased; it is may be attributed to increasing the hydrophilicity of the molecules, leading to increase solvation due to hydrogen bonding.

Table VI

Surface properties of the prepared surfactants (ix-x) $\mathrm{R}-\mathrm{CO}-\mathrm{NH}\left(-\mathrm{CH}_{2}-\mathrm{CH}_{2} \mathrm{NH}-\right)_{n}-\mathrm{CH}_{2}-\mathrm{CH}(\mathrm{OH})-\mathrm{CH}_{2}-\mathrm{NEt}_{3}^{+} \mathrm{OH}^{-}$

\begin{tabular}{|c|c|c|c|c|c|c|c|c|c|c|}
\hline \multirow{2}{*}{\multicolumn{2}{|c|}{ Compd. no. }} & \multirow{3}{*}{$\begin{array}{l}\mathrm{S} . \mathrm{T} \\
\mathrm{mN} / \mathrm{m} \\
(0.1 \%)\end{array}$} & \multirow{3}{*}{$\begin{array}{l}\text { I.F.T. } \\
\text { mNNm } \\
(0.1 \%)\end{array}$} & \multirow{3}{*}{$\begin{array}{c}\text { Cloud point } \\
{ }^{\circ} \mathrm{C} \\
(1 \%)\end{array}$} & \multirow{3}{*}{$\begin{array}{l}\text { Wetting time } \\
(\text { sec.) } \\
(0.1 \%)\end{array}$} & \multirow{3}{*}{$\begin{array}{l}\text { Foam height } \\
(\mathrm{m} . \mathrm{m} .) \\
(0.1 \%)\end{array}$} & \multicolumn{2}{|c|}{ Emul. stability } & \multirow{2}{*}{\multicolumn{2}{|c|}{$\frac{\text { Stability to hydrolysis }}{\text { Base }}$}} \\
\hline & & & & & & & \multirow[b]{2}{*}{ (min.) } & \multirow[b]{2}{*}{ (sec.) } & & \\
\hline & & & & & & & & & (min.) & (sec.) \\
\hline \multirow[t]{4}{*}{$C_{16}$} & $i x_{a}$ & 42.5 & 9.5 & - & 68 & 205 & 234 & 40 & 498 & 13 \\
\hline & $i x_{b}$ & 41.0 & 8.0 & 95 & 62 & 250 & 372 & 43 & 681 & 29 \\
\hline & $i x_{c}$ & 38.0 & 7.5 & $>100$ & 35 & 350 & 401 & 37 & 710 & 36 \\
\hline & $i x_{d}$ & 36.5 & 5.0 & $>100$ & 28 & 510 & 518 & 53 & 772 & 32 \\
\hline \multirow[t]{4}{*}{$\mathrm{C}_{18}$} & $x_{a}$ & 44.0 & 12 & - & 76 & 230 & 267 & 42 & 431 & 43 \\
\hline & $x_{b}$ & 43.5 & 9.0 & 91 & 68 & 260 & 391 & 29 & 630 & 56 \\
\hline & $x_{c}$ & 41.5 & 8.0 & $>100$ & 54 & 310 & 429 & 25 & 732 & 38 \\
\hline & $x_{d}$ & 39.0 & 6.5 & $>100$ & 47 & 480 & 543 & 19 & 787 & 20 \\
\hline
\end{tabular}


Table VII

Surface properties of the prepared surfactants (ix-xii)a-d $\mathrm{R}-\mathrm{CO}-\mathrm{NH}\left(-\mathrm{CH}_{2}-\mathrm{CH}_{2} \mathrm{NH}-\right)_{n}-\mathrm{CH}_{2}-\mathrm{CH}(\mathrm{OH})-\mathrm{CH}_{2}-\mathrm{NEt}^{+}{ }_{3} \mathrm{OH}^{-}$

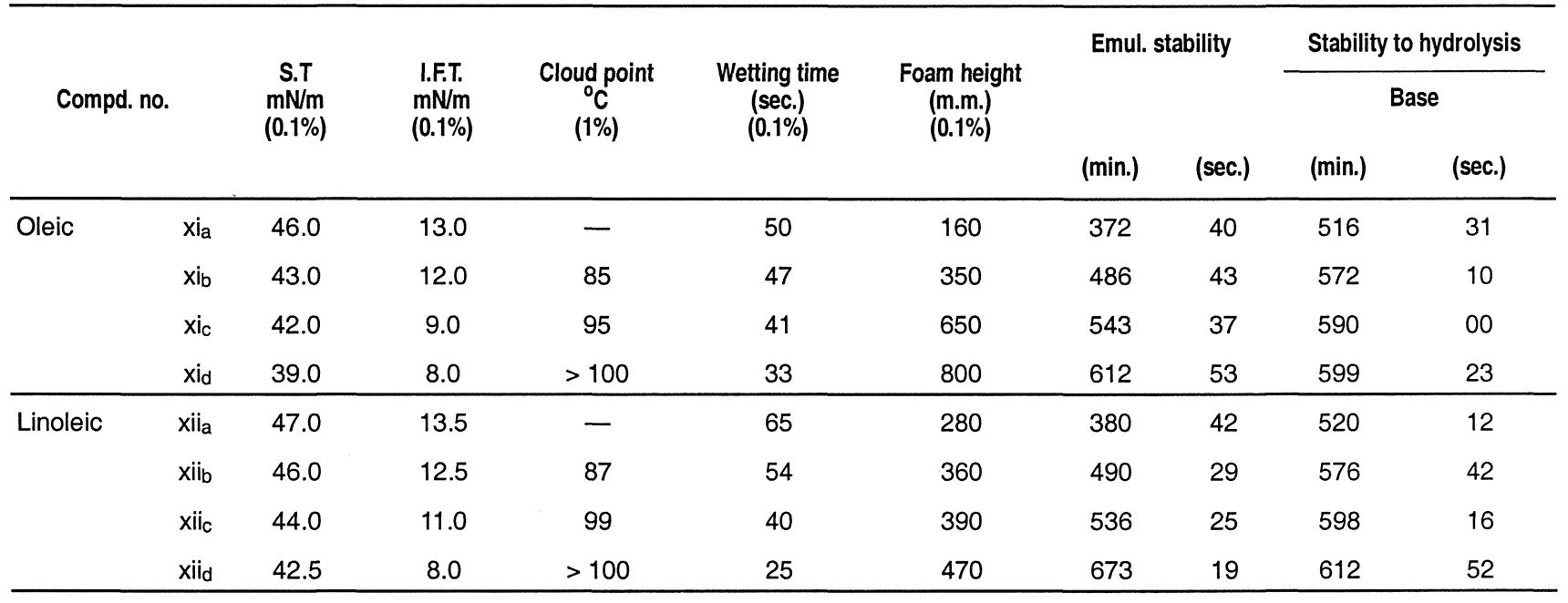

Table VIII

Surface properties of the mixed cationic surfactants prepared from rice bran oil (xiii)a-d $\mathrm{R}-\mathrm{CO}-\mathrm{NH}\left(-\mathrm{CH}_{2}-\mathrm{CH}_{2} \mathrm{NH}-\right)_{n}-\mathrm{CH}_{2}-\mathrm{CH}(\mathrm{OH})-\mathrm{CH}_{2}-\mathrm{NEt}_{3}^{+} \mathrm{OH}^{-}$

\begin{tabular}{|c|c|c|c|c|c|c|c|c|c|}
\hline \multirow{3}{*}{ Compd. no. } & \multirow{3}{*}{$\begin{array}{c}\mathrm{S} . \mathrm{T} \\
\mathrm{mN} / \mathrm{m} \\
(0.1 \%)\end{array}$} & \multirow{3}{*}{$\begin{array}{l}\text { I.FT. } \\
\mathrm{mNNm} \\
(0.1 \%)\end{array}$} & \multirow{3}{*}{$\begin{array}{c}\text { Cloud point } \\
{ }_{0} \mathrm{C} \\
(1 \%)\end{array}$} & \multirow{3}{*}{$\begin{array}{l}\text { Wetting time } \\
(\text { sec.) } \\
(0.1 \%)\end{array}$} & \multirow{3}{*}{$\begin{array}{l}\text { Foam height } \\
\left(\begin{array}{l}\text { m.m.) } \\
(0.1 \%)\end{array}\right.\end{array}$} & \multicolumn{2}{|c|}{ Emul. stability } & \multirow{2}{*}{\multicolumn{2}{|c|}{$\begin{array}{c}\text { Stability to hydrolysis } \\
\text { Base }\end{array}$}} \\
\hline & & & & & & & & & \\
\hline & & & & & & (min.) & (sec.) & (min.) & (sec.) \\
\hline xiiia & 50.0 & 13.5 & - & 35 & 290 & 370 & 40 & 473 & 58 \\
\hline xiiib & 48.0 & 12.0 & $>100$ & 28 & 350 & 395 & 43 & 498 & 45 \\
\hline xiiic & 43.5 & 8.5 & $>100$ & 20 & 420 & 412 & 37 & 513 & 33 \\
\hline xiild & 40.5 & 6.0 & $>100$ & 10 & 530 & 501 & 53 & 521 & 39 \\
\hline
\end{tabular}

3.2. Kraft and cloud points: All these cationic surfactants (ix-xiii) $)_{\text {a-d }}$ were readily soluble in water, and have excellent hydrophilicity for practical use because their kraft points $\left(T_{\mathrm{kp}}\right)$ were below $0{ }^{\circ} \mathrm{C}$, and their high cloud point (cf. Tables VI-VIII); it is considered that, the remarkable decrease in $\left(T_{\mathrm{kp}}\right)$ and high cloud point for the prepared surfactants is attributed to the co-operative effect of quaternary ammonium moiety and repeating nitrogen linkages.

3.3. Foam height and wetting: It is reported that, the efficiency of surfactant as a foamer increases with increased alkyl chain length (20-21). In general the foam height and wetting time for the surfactants prepared from saturated fatty acids (ix- $\mathrm{x})_{\mathrm{a}-\mathrm{d}}$ recorded more improving than those prepared from unsaturated acids (xi-xii) $)_{a-d}$. On the other hand, cationic surfactants (xiii) $)_{a-d}$ prepared from mixed fatty acids of (RBO) revealed good wetting properties.
3.4. Emulsion stability: Studies are still being carried out on the utilization of surfactants in emulsion formation which is of immense importance to technological development. It was proved that the emulsifying stability of the prepared surfactants especially those of (RBO) exhibit good emulsifying properties (cf. Tables VI-VIII) which make them of choice in pesticide and cosmetic formulations.

3.5. Stability towards acid and base: Concerning to the stability towards acid and base hydrolysis, all the prepared surfactants have higher stability in acidic than in basic medium (cf. Tables VI-VIII); this may be due to the presence of free nitrogen atom which reacted with the acid forming more cationic centers (22).

3.6. Corrosion Inhibition: This was done by using weight loss technique. Table (IX) shows that the effect of increasing concentrations of the prepared 
cationic surfactants (as example $x_{d}$, xiii $i_{d}$ ) on corrosion of carbon steel specimen in $3.5 \% \mathrm{NaCl}$ as corrosion medium. It reveals that, the weight loss decreases as the concentration of the tested examples increases and hence increases the value of inhibition efficiency. Also, it was found that, the cationic surfactant prepared from mixed fatty acids of rice bran oil (xiii), recorded higher inhibition efficiency than $(x)_{d}$. These effected was attributed to their adsorption and the formation of a barrier film on the metal surface (23).

Table IX

\section{Effect of increasing concentrations of cationic surfactants on the weight loss of carbon steel in $3.5 \% \mathrm{NaCl}$ solution}

\begin{tabular}{|c|c|c|c|}
\hline \multicolumn{2}{|c|}{$\begin{array}{l}\text { Inhibition concentration } \\
(\mathrm{ppm})\end{array}$} & \multirow{2}{*}{$\begin{array}{c}\begin{array}{c}\text { Weight loss } \\
\left(\mathrm{mg} \mathrm{dm}^{-2}\right)\end{array} \\
850\end{array}$} & \multirow{2}{*}{$\begin{array}{c}\begin{array}{c}\text { Inhibition } \\
\text { efficiency }\end{array} \\
-\end{array}$} \\
\hline$x d$ & 00.00 & & \\
\hline & 10.00 & 640 & 24.70 \\
\hline & 50.00 & 560 & 34.11 \\
\hline & 100.00 & 512 & 39.76 \\
\hline & 200.00 & 435 & 48.82 \\
\hline & 300.00 & 315 & 62.94 \\
\hline & 500.00 & 105 & 87.64 \\
\hline \multirow[t]{7}{*}{ xiiild } & 00.00 & 850 & - \\
\hline & 10.00 & 614 & 27.76 \\
\hline & 50.00 & 535 & 37.05 \\
\hline & 100.00 & 466 & 45.17 \\
\hline & 200.00 & 395 & 53.52 \\
\hline & 300.00 & 285 & 66.47 \\
\hline & 500.00 & 98 & 88.47 \\
\hline
\end{tabular}

\section{CONCLUSION}

From the previous results, may conclude that: All the prepared cationic surfactants have good surface properties, and they could be used as good emulsifier in non edible medium as insecticides, pesticides and also as corrosion inhibitors. The local non edible crude rice bran oil can be used as raw material for preparing these surfactants.

\section{BIBLIOGRAPHY}

1. Muzycsko, T. M., Shore, S. and Loboda, J. A. (1968).«Fatty amidoamine derivatives: N,N-dimethyl-N-(3- alkylamidopropyl) amines and their salts».-J. Am. Oi Chem. Soc. 45, 720-725.

2. Yamamoto. T., Sumida, S. and Nanba, Y. (1963)."Synthesis and properties of surface active agents. VII. Studies on cationic surfactants as inhibitors for acid-cleaning of metal».-Yukagaku 12, 365. C. A. 59: $15502 \mathrm{~g}$ (1963).

3. Komkov, I. P. and Pankratov, V. A. (1970).-«Zh. Prik Khim».-43 (6). C.A. 73: 100287 (1970).

4. Oreal, S.A. (1966).- «Netherlands Patent».-6, 604 104. C.A. 66: 57029h (1967).

5. Olsson, E. (1963)._Finska Kemists Medd. 72. N. ${ }^{\circ} 4$.

6. Aoya, Hiroshi., Kadowaki, Minoru., Hosaka Shuntaro, Nishie.-Takamitsu Japan patent 73. 15. 210.

7. Devinsky, F., Lacko, I., Mlynarcik, D., Racansky, V. and Krasnec (1985).— «Relationship between critical micelle concentrations and minimum inhibitory concentrations for some non-aromatic; quaternary ammonium salts and amine oxides".-Tens. Det. 22, 10-15.

8. Yu-Lin Jiang., Yu Qiao Hu., Jun Pang and Yun-Cheng Yuan (1996). - «Synthesis of long-chain quaternary ammonium salts from alcohols by microwave irradiation".-J. Am. Oil. Chem. Soc. 73, (7) 847-850.

9. El-Sawy, A. A. (1989).- «Synthesis of sucrose esters from rice bran oil fatty acids".-Grasas y Aceites 40, 382-384.

10. Gawish, S. M., Hazzaa, A. A. B. and Gebril El-Din, B. (1978).- «Cationic surface active agents».-J. Am. Oil. Chem. Soc. 55, 745-747.

11. Jungermann. E. (1970)._-Cationic surfactants».-Vol. 4 Marcel Dekker Inc., New York.

12. Findly, A. (1963).- «Practical physical chemistry»Longmans, London, $6^{\text {th }}$. Ed. 1040.

13. Wiel, J. K., Smith, F. D., Stirton, A. J. and Bistline, R. G Jr. (1963). - «Long chain alkanesulfonates and 1-hydroxy-2- alkanesulfonates: Structure and property relations". - J. Am. Oil. Chem. Soc. 40, 538-540.

14. Durham, K. (1961)._- «Surface activity and detergency».MacMillan \& Co. Ltd., London.

15. Draves, C. Z. and Clarkso, R. (1931).-J. Am. Dye Stuf Reporter, 20, 201.

16. Ross, $j$. and Miles, G.D. (1941).- «Apparatus for comparison of foaming properties ot soaps and detergents".-Oil and Soap 18, 99.

17. Takeshi H. (1970).-Bull. Chem. Soc. 43, 2236 (Japan).

18. El-Sukkary, M. M. A., El-Sawy, A. A. and El-Dib, F. (1987).-Hungarian J. of Industrial Chem. 15, 417.

19. Evans. U. R. (1960).- - «The corrosion and oxidation of metals".-Arnold, London, 135.

20. Seong, K. T., Toshiyuki, K., Yohji, N., Toshikazu, H. and Isoa Ikeda. (1996). - «Surface-active properties of novel cationic surfactants with two ammonio groups".J. Am. Oil. Chem. Soc. 73 (7), 907-911.

21. Rosen, M. J. (1989). - «Surfactants and interfacial phenomena" $2^{\text {nd }}$ Ed., John Wiley \& Sons, New York. 286-294.

22. Eissa, A. M. F., Mahmoud, A. A. and El-Sawy, A.A. (1993).«Preparation and surface active properties of oxypropylated diol monoesters of fatty acids with an amide oxime terminal group".-Grasas y Aceites 45, 306-308.

23. Tedeschi, R. J. (1975).-Corrosion 31, 136. 